\title{
The one-loop analysis of the beta-function in the Schroedinger Functional for Moebius Domain Wall Fermions
}

\section{Yuko Murakami*}

Graduate school of Science, Hiroshima University, Higashi-Hiroshima, Hiroshima 739-8526, Japan

E-mail: vukomhiregmail.com

\section{Ken-Ichi Ishikawa}

Graduate school of Science, Hiroshima University, Higashi-Hiroshima, Hiroshima 739-8526,

Japan,

Core of Research for Energetic Universe, Hiroshima University, Higashi-Hiroshima, Hiroshima

739-8526, Japan

E-mail: ishikawaetheo.phys.sci.hiroshima-u.ac.ip

\begin{abstract}
We proposed a construction of the Schroedinger functional scheme for the Moebius domain wall fermions (MDWF) by introducing a proper boundary operator to the original MDWF in the last conference. The spectrum of the effective four-dimensional operator was investigated. This year we investigate the fermionic contribution to the beta-function with the Moebius domain wall fermion with the SF boundary term up to the one-loop level and find that our construction properly reproduce the one-loop beta-function.
\end{abstract}

The 33rd International Symposium on Lattice Field Theory

14 -18 July 2015

Kobe International Conference Center, Kobe, Japan*

\footnotetext{
* Speaker.
} 


\section{Introduction}

The chiral symmetry is important not only in QCD but also in the standard model. To extract hadronic observables based on the chiral symmetry, we need both the lattice field theory and the chiral symmetry. The lattice chiral symmetry is now realized by the overlap and domain wall fermions and extensive efforts have been devoted to the accurate values for hadronic observables sensitive to the chiral symmetry[四, []. In this sense the non-preturbative renormalization for the lattice fermions with the lattice chiral symmetry becomes important.

The Schroedinger functional (SF) scheme [3] has been successfully applied to renormalize the lattice QCD with the Wilson fermions non-perturbatively [䧃]. Although it was not simple for the lattice chiral fermions to apply the SF scheme, the SF methods for the lattice chiral fermions have

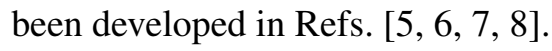

In the last lattice conference, we proposed a construction of the SF scheme for Moebius domain wall fermions (MDWF) [Q]. The operator is modified by adding a temporal boundary operator based on Takeda's implementation for the standard domain wall fermion [8]. The form of the boundary operator is determined by the symmetry argument of Ref. [5] so that the term holds the discrete symmetries ( $C, P, T, \Gamma_{5}$-Hermiticity) and breaks the domain wall chiral symmetry [ए]] at the temporal boundary. We investigated the properties of the effective four-dimensional operator induced from the MDWF with the boundary operator and found that it reproduced the proper continuum theory with the SF boundary only in the infinite fifth lattice extent $N_{5} \rightarrow \infty$.

In this paper, we extend our previous work to obtain the proper operator for the SF scheme even at a finite $N_{5}$. To do this we properly renormalize the effective four-dimensional operator since the explicit breaking of the lattice chiral symmetry at a finite $N_{5}$ induces the additive residual mass [W] as seen in Wilson type fermions even at the tree-level. After renormalizing the effective four-dimensional operator we investigate the eigenvalues in the SF boundary condition. In order to check the consistency of our construction, we also investigate the fermionic contribution to the one-loop beta-function in the SF scheme at a finite $N_{5}$.

This paper is organized as follows. In the next section, we briefly explain the renormalization factors for the MDWF at the tree-level without the SF boundary condition. The renormalization factors are applied to the case of the SF scheme. Then we introduce the MDWF with the SF boundary term given by our previous work [Q] The lowest eigenvalues of the effective four-dimensional operator are investigated. In section [1, we confirm that the properly renormalized MDWF operator with the SF boundary term reproduces the proper one-loop beta-function. The scaling violation on the step scaling function is also examined at the one-loop level. We summarize this work in the last section.

\section{The set up of the SF scheme for the MDWF}

The Moebius domain wall fermion (MDWF) [ㅁ] is one of the lattice chiral fermion and 
defined as the fifth dimensional operator as follows.

$$
\begin{aligned}
D_{\mathrm{MDWF}}(n, s ; m, t) & =D_{s}^{+}(n ; m) \delta_{s, t}+D_{s}^{-}(n ; m) M_{5}(s ; t), \\
D_{s}^{+}(n ; m) & =b_{s} D_{\mathrm{WF}}(n ; m)+1, \\
D_{s}^{-}(n ; m) & =c_{s} D_{\mathrm{WF}}(n ; m)-1, \quad\left(s=1, \cdots, N_{5}\right) \\
M_{5}(s ; t) & =P_{L} \delta_{s+1, t}+P_{R} \delta_{s-1, t}-m_{f}\left[P_{L} \delta_{s, t} \delta_{1, t}+P_{R} \delta_{s, 1} \delta_{N_{5}, t}\right],
\end{aligned}
$$

where $(s, t)$ is the lattice index in the fifth direction, $(n, m)$ is the four-dimensional lattice site index, $m_{f}$ is the mass parameter, $D_{W F}$ is the Wilson fermion operator with a negative mass $\left(m_{0}\right), P_{R / L}$ is the chiral projection: $P_{R / L}=\left(1 \pm \gamma_{5}\right) / 2,\left(b_{s}, c_{s}\right)$ are the Moebius parameters, and $N_{5}$ is the lattice size in the fifth direction. The MDWF is a generalization of the domain wall fermions and includes

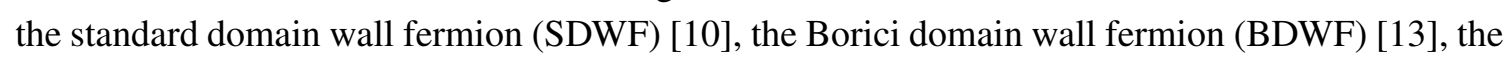
optimal Shamir domain wall fermion [U4] and the optimal Chiu domain wall fermion (CDWF) [L5] by adjusting $\left(b_{s}, c_{s}\right)$. The Moebius parameters for the optimal type domain wall fermions can be derived from the Zolotarev sign function approximation [ए6].

The effective four-dimensional operator calculated from the MDWF operator yields the overlap fermion operator [17, [8]] at the infinite extent of the fifth direction [10, [20] $\left(N_{5} \rightarrow \infty\right)$. At a finite $N_{5}$ the effective operator does not satisfy the Ginsparg-Wilson relation [2] and an $O(a)$ error is expected, so that the relation to the pole mass and the bare mass $m_{f}$ can be differ in this case. The effective four-dimensional operator, $D_{\text {eff }}^{\left(N_{5}\right)}$, at a finite $N_{5}$ without the SF boundary condition is evaluated as

$$
\begin{aligned}
a D_{\mathrm{eff}}^{\left(N_{5}\right)} & \equiv P^{T} D_{\mathrm{PV}}^{-1} D_{\mathrm{MDWF}}\left(a m_{f}\right) P \\
& =\frac{1+a m_{f}}{2}-\frac{1-a m_{f}}{2} \gamma_{5} R_{N_{5}}\left(\mathscr{H}_{W}\right), \\
R_{N_{5}}\left(\mathscr{H}_{\omega}\right) & =\frac{\prod_{s=1}^{N_{5}}\left(1+\omega_{s} \mathscr{H}_{W}\right)-\prod_{s=1}^{N_{5}}\left(1-\omega_{s} \mathscr{H}_{W}\right)}{\prod_{s=1}^{N_{5}}\left(1+\omega_{s} \mathscr{H}_{W}\right)+\prod_{s=1}^{N_{5}}\left(1-\omega_{s} \mathscr{H}_{W}\right)} \\
\mathscr{H}_{W} & =\frac{a D_{W F}}{a_{5} D_{W F}+2}, \\
a_{5} & =b_{s}-c_{s}, \quad \omega_{s}=b_{s}+c_{s}, \\
P(s) & =P_{L} \delta_{s, 1}+P_{R} \delta_{s, N_{5}},
\end{aligned}
$$

where $D_{\mathrm{PV}}=D_{\mathrm{MDWF}}\left(a m_{f}=1.0\right)$. The coefficients $a_{5}$ and $\omega_{s}$ must be tuned to properly reproduce the sign function as $R_{N_{5}}(x) \rightarrow \operatorname{sign}(x)$ in the limit of $N_{5} \rightarrow \infty$. The ordering of $\left(b_{s}, c_{s}\right)$ in the fifth direction is irrelevant for the effective four-dimensional operator without the SF boundary condition. In the continuum limit the effective four-dimensional operator behaves as

$$
\begin{aligned}
a D_{e f f}^{\left(N_{5}\right)} & \approx Z_{N_{5}}\left(a \not D+a m_{\mathrm{res}}\right), \\
Z_{N_{5}} & =\frac{\left(1-a m_{f}\right) R_{N_{5}}(\alpha)}{\left(a m_{0}\right)\left(2-\left(a m_{0}\right) a_{5}\right)}, \\
a m_{\mathrm{res}} & =\left[\frac{1+a m_{f}}{1-a m_{f}} \frac{1}{R_{N_{5}}(\alpha)}-1\right] \frac{\left(a m_{0}\right)\left(2-\left(a m_{0}\right) a_{5}\right)}{2}, \\
\alpha & =\frac{\left(a m_{0}\right)}{\left(2-\left(a m_{0}\right) a_{5}\right)},
\end{aligned}
$$


where $\not D$ is the Dirac operator in the continuum, $Z_{N_{5}}$ is the normalization factor and $m_{\text {res }}$ is the residual mass at the tree-level []]]. Even at $m_{f}=0$ the residual mass does not vanish as $R_{N_{5}}(\alpha) \neq 1$ at a finite $N_{5}<\infty$. In order to investigate the property of the effective operator at the vanishing pole mass, we renormalize the effective operator as follows,

$$
a D_{R}^{\left(N_{5}\right)}=Z_{N_{5}}^{-1} a D_{e f f}^{\left(N_{5}\right)}\left(a m_{\mathrm{cr}}\right), \quad \text { where } \quad a m_{\mathrm{cr}}=\frac{R_{N_{5}}(\alpha)-1}{R_{N_{5}}(\alpha)+1} .
$$

Now we consider the effective four-dimensional operator in the SF scheme. In our previous work, we proposed the following operator for the MDWF in the SF scheme [Q] ].

$$
\begin{aligned}
D_{\mathrm{MDWF}}^{\mathrm{SF}}(n, s ; m, t) & =D_{\mathrm{MDWF}}(n, s ; m, t)+c_{\mathrm{SF}} B_{\mathrm{SF}}(n, s ; m, t), \\
B_{S F}(n, s ; m, t) & =f(s) \delta_{s, N_{5}-t+1} \delta_{n, m} \delta_{n_{4}, m_{4}} \gamma_{5}\left(\delta_{n_{4}, 1} P_{-}+\delta_{n_{4}, T-1} P_{+}\right), \\
f(s) & =\left\{\begin{array}{ll}
-1 & \left(\text { for } 1 \leq s \leq N_{5} / 2\right) \\
+1 & \left(\text { for } N_{5} / 2+1 \leq s \leq N_{5}\right)
\end{array},\right.
\end{aligned}
$$

where $P_{ \pm}$is the projection: $P_{ \pm}=\left(1 \pm \gamma_{4}\right) / 2$. We introduce the boundary operator $B_{\mathrm{SF}}$ to satisfy the SF boundary condition, which explicitly breaks the domain wall chiral symmetry [ए0] at the temporal boundary, as suggested in Refs. [[5], [8]. In order to keep the discrete symmetries $C, P, T$, and $\Gamma_{5}$-Hermiticity we require the parity symmetry in the fifth direction because the ordering of the coefficients $\left(b_{s}, c_{s}\right)$ in the fifth direction is relevant in this case. A quasi optimal choice for $\omega_{s}$ with the parity symmetry are determined according to Ref. [Q]. We call the domain wall fermion operators with the quasi optimal choice for $\omega_{s}$ having the parity symmetry in the fifth direction as the palindromic Shamir domain wall fermion (PSDWF) and the palindromic Chiu domain wall fermion (PCDWF) depending on the choice for the kernel operator. We use the same form as Eq. (2.14) for the normalization factor and the pole mass with the SF boundary condition, though we cannot obtain the effective four-dimensional operator in a simple closed form like Eq. (2.5) and the corresponding behavior in the continuum limit like Eq. (2.10).

We investigate the lowest eigenvalues of the effective four-dimensional operator derived from Eq. (2.15) at the tree-level. We use $L=T$ and the standard boundary condition for the gauge field [四] yielding a constant background chromoelectric field. The generalized periodic boundary condition with the phase angle $\theta=\pi / 5$ is used for the spatial boundary condition. In order to study the effect of the renormalization with a finite fifth dimensional extent at the tree-level, we set $m_{0}=1.5$ and $\left(b_{s}, c_{s}\right)=(1.0,0.0)$ for the SDWF and $m_{0}=1.5$ and $\left(b_{s}, c_{s}\right)=(1.0,1.0)$ for the BDWF and $m_{0}=$

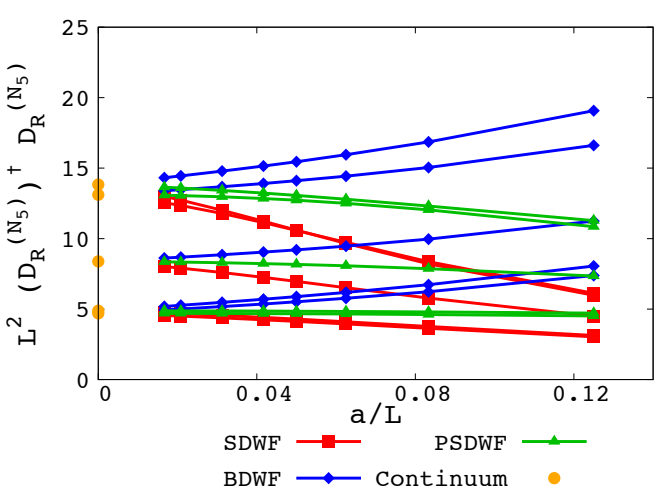

Figure 1: The scaling behavior of the lowest five eigenvalues of the Hermitian squared effective four-dimensional operator. 1.0 for the PSDWF at $N_{5}=8$ and $c_{S F}=1.0$.

Figure $\square$ shows the lowest five eigenvalues of the Hermitian squared effective four-dimensional operator $L^{2}\left(D_{R}^{\left(N_{5}\right)}\right)^{\dagger} D_{R}^{\left(N_{5}\right)}$ for each operator type. The yellow dot points at the continuum limit 
are quoted from Ref [22]. We confirm that the operator involving the tree-level renormalization properly reproduces the continuum theory.

\section{The one-loop beta-function}

We calculate the fermoinic contribution to the one-loop beta-function using the MDWF with the SF boundary condition. The renormalized coupling constant in the SF scheme [3] is defined by

$$
\frac{1}{g_{\mathrm{SF}}^{2}}=\left.\frac{1}{k} \frac{\partial \Gamma}{\partial \eta}\right|_{\eta=v=0}
$$

where $\Gamma$ is the effective action with the SF boundary condition, $\eta$ and $v$ are parameters for the SF boundary condition, and $k$ is a normalization factor based on the tree-level analysis. For the MDWF in the SF scheme, the fermionic contribution to the one-loop beta-function can be obtained from the following term;

$$
p_{1,1}=\left.\frac{1}{k} \frac{\partial \Gamma_{1}}{\partial \eta}\right|_{\eta=v=0}=\left.\frac{1}{k} \frac{\partial}{\partial \eta}\left[\ln \operatorname{det}\left(D_{\mathrm{MDWF}}^{\mathrm{SF}}\left(D_{\mathrm{PV}}^{\mathrm{SF}}\right)^{-1}\right)\right]\right|_{\eta=v=0},
$$

where $\Gamma_{1}$ is the one-loop effective action of the fermion part and $D_{\mathrm{PV}}^{\mathrm{SF}}=D_{\mathrm{MDWF}}^{\mathrm{SF}}\left(a m_{f}=1.0\right)$. The cut-off dependence of $p_{1,1}$ can be written in the following form asymptotically;

$$
p_{1,1}=\sum_{n=0}(a / L)^{n}\left[A_{n}+B_{n} \ln (L / a)\right]=A_{0}+B_{0} \ln (L / a)+(a / L)\left(A_{1}+B_{1} \ln (L / a)\right)+O\left(a^{2}\right) .
$$

$B_{0}$ contains the information of the fermionic contribution to the one-loop beta-function and it should be $B_{0}=2 b_{0,1}=-1 /\left(12 \pi^{2}\right)=-0.008443 \ldots$. We numerically calculate $p_{1,1}$ using the MDWF with the SF boundary of Eq. (2.15) and the proper renormalization at the vanishing pole mass, and fit them with the function Eq. (B.3]) up to $O(a)$. Table $\mathbb{W}$ shows the result of the fitting. The values for $c_{\mathrm{SF}}$ are determined with the $O(a)$ improvement procedure based on the PCAC relation [23] at the tree-level. We find that $B_{0}$ are consistent with the $2 b_{0,1}=-0.008443 \ldots$ within the fitting error for each DWF type and confirm that the MDWF with the SF boundary term we constructed at the tree-level properly reproduces with the fermionic contribution to the one-loop beta-function.

\begin{tabular}{|c|c|c|c|c|c|}
\hline Operator & $m_{0}$ & $c_{S F}$ & $N_{5}$ & Fit range $\left[L_{\min } / a, L_{\max } / a\right]$ & $B_{0}\left(\times 10^{-3}\right)$ \\
\hline SDWF & 1.5 & 0.520 & 8 & {$[18: 48]$} & $-8.43 \pm 0.01$ \\
\cline { 4 - 6 } & & & 16 & {$[26: 48]$} & $-8.441 \pm 0.004$ \\
\hline \multirow{2}{*}{ BDWF } & \multirow{2}{*}{1.5} & 0.312 & 8 & {$[18: 48]$} & $-8.37 \pm 0.08$ \\
\cline { 4 - 6 } & & & 16 & {$[34: 48]$} & $-8.46 \pm 0.05$ \\
\hline \multirow{2}{*}{ PSDWF } & \multirow{2}{*}{1.0} & 0.820 & 8 & {$[10: 48]$} & $-8.2 \pm 0.3$ \\
\cline { 3 - 6 } & & 0.630 & 16 & {$[16: 48]$} & $-8.44 \pm 0.03$ \\
\hline
\end{tabular}

Table 1: The fit results for $B_{0}$.

We investigate the lattice cut-off dependence for the step scaling function (SSF) [24]. The deviation of the SSF at a finite cut-off from that in the continuum limit is defined by

$$
\delta=\frac{\Sigma(s, u, a / L)-\sigma(s, u)}{\sigma(s, u)}=\delta_{1} u+\delta_{2} u^{2}+\cdots
$$




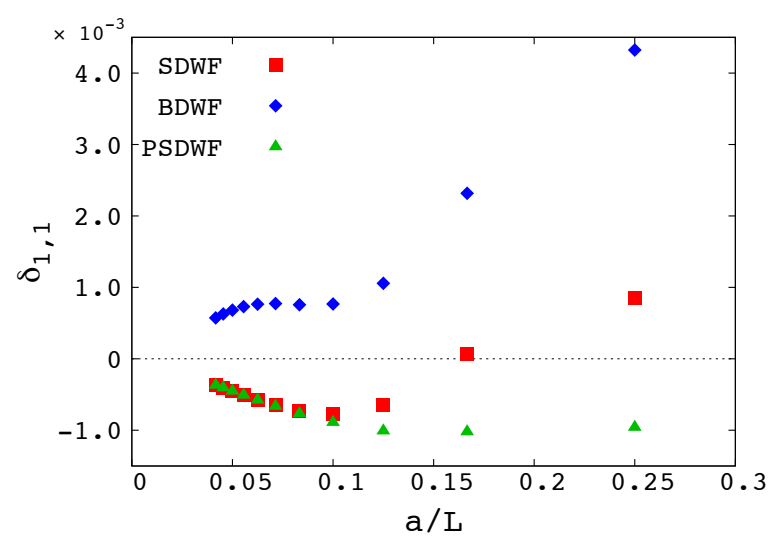

Figure 2: The deviation of the SSF between the lattice and the continuum theory as a function of the lattice spacing. $\left(N_{5}=16,2 b_{0,1}=-1 /\left(12 \pi^{2}\right)\right)$

where $u$ is the SF scheme coupling constant $u=g_{\mathrm{SF}}^{2}(L)$ renormalized at $L, \Sigma(s, u, a / L)=g_{\mathrm{SF}}^{2}(s L)$ is the SSF at a finite lattice cut-off, $\sigma(s, u)=g_{\mathrm{SF}}^{2}(s L)$ is that in the continuum theory, and $s$ is a scale factor. $\delta$ can be expanded as a polynomial of $u$ perturbatively as shown in the equation. $\delta_{1}$ must vanish in the continuum limit at the one-loop level analysis. The fermionic contribution to $\delta_{1}$ is given by

$$
\begin{aligned}
\delta_{1} & =\delta_{1,0}+\delta_{1,1} n_{f}, \\
\delta_{1,1} & =p_{1,1}(a /(2 L))-p_{1,1}(a / L)-2 b_{0,1} \ln 2,
\end{aligned}
$$

where we set $s=2$.

The lattice cut-off dependence of Eq. (3.6) with the MDWF at $N_{5}=16$ is shown in Figure $\square$, where $2 b_{0,1}=-1 /\left(12 \pi^{2}\right)$ is imposed. Although rather complicated cut-off dependence of $\delta_{1,1}$ is seen, it goes to vanish in the continuum limit. Therefore we conclude that the renormalized MDWF with the SF scheme is consistent with the continuum massless Dirac operator at the one-loop level. The cut-off dependence includes the $O(a)$ error from $A_{1}$ and $B_{1}$ terms of Eq. (B.3]). The former can be removed by tuning the boundary operator of the gauge action, while the latter requires the bulk $O(a)$-improvement term such as the clover term for the Wilson fermion because the lattice chiral symmetry is broken explicitly at a finite $N_{5}$.

\section{Summary}

We investigated the Schroedinger Functional (SF) scheme with the Moebius domain wall fermions (MDWF). The MDWF with the SF boundary term was introduced and the spectrum of the massless Dirac operator with the SF boundary condition is reproduced from the MDWF at the tree-level after applying the proper renormalization even at a finite fifth dimensional extent. We also confirmed that the MDWF operator with the SF boundary term reproduced the fermionic part of the universal one-loop beta-function in the SF scheme. From these analysis at the one-loop level, we expect that the SF scheme is applicable to the MDWF at a finite $N_{5}$, which can be regarded as a kind of better Wilson fermions, by adding the SF boundary term. 
We will check the consistency of the step scaling function of the coupling calculated from the MDWF with the SF boundary term non-perturbatively in the future work.

\section{Acknowledgment}

We thank to S. Takeda for the helpful advice. A part of numerical computations is performed on the INSAM (Institute for Numerical Simulations and Applied Mathematics) GPU Cluster at Hiroshima University. This work was supported in part by a Giant-in-Aid for Scientific Research (C) (No. 24540276) from the Japan Society for the Promotion of Science (JSPS) and the MEXT program for promoting the enhancement of research universities, Japan.

\section{References}

[1] J. M. Flynn, T. Izubuchi, T. Kawanai, C. Lehner, A. Soni, R. S. Van de Water and O. Witzel, Phys. Rev. D 91, no. 7, 074510 (2015) [arXiv:1501.05373 [hep-lat]].

[2] S. Aoki et al., Eur. Phys. J. C 74, 2890 (2014) [arXiv:1310.8555 [hep-lat]].

[3] M. Lüscher, Nucl. Phys. B254, 52 (1985)

[4] M. Luscher, R. Sommer, P. Weisz and U. Wolff, Nucl. Phys. B 413, 481 (1994) [hep-lat/9309005].

[5] M. Luscher, JHEP 0605, 042 (2006) [hep-lat/0603029].

[6] Y. Taniguchi, JHEP 0512, 037 (2005) [hep-lat/0412024].

[7] Y. Taniguchi, JHEP 0610, 027 (2006) [hep-lat/0604002].

[8] S. Takeda, Phys. Rev. D 87, no. 11, 114506 (2013) [arXiv:1010.3504 [hep-lat]].

[9] Y. Murakami and K. I. Ishikawa, PoS LATTICE 2014, 331 (2014) [arXiv:1410.8335 [hep-lat]].

[10] V. Furman and Y. Shamir, Nucl. Phys. B 439, 54 (1995) [hep-lat/9405004].

[11] S. Capitani, PoS LAT 2007, 066 (2007) [arXiv:0708.3281 [hep-lat]].

[12] R. C. Brower, H. Neff and K. Orginos, Nucl. Phys. Proc. Suppl. 140, 686 (2005) [hep-lat/0409118].

[13] A. Borici, hep-lat/9912040.

[14] A. Borici, hep-lat/0211001.

[15] T. W. Chiu, Phys. Rev. Lett. 90, 071601 (2003) [hep-lat/0209153].

[16] T. W. Chiu, T. H. Hsieh, C. H. Huang and T. R. Huang, Phys. Rev. D 66, 114502 (2002) [hep-lat/0206007].

[17] R. Narayanan and H. Neuberger, Nucl. Phys. B 443, 305 (1995) [hep-th/9411108].

[18] H. Neuberger, Phys. Lett. B 417 (1998) 141 [hep-lat/9707022].

[19] Y. Kikukawa and T. Noguchi, doi:10.1016/S0920-5632(00)91758-4 hep-lat/9902022.

[20] A. Borici, hep-lat/0402035.

[21] P. H. Ginsparg and K. G. Wilson, Phys. Rev. D 25, 2649 (1982).

[22] S. Sint and R. Sommer, Nucl. Phys. B 465, 71 (1996) [hep-lat/9508012].

[23] M. Luscher, S. Sint, R. Sommer and P. Weisz, Nucl. Phys. B 478, 365 (1996) [hep-lat/9605038].

[24] M. Luscher, P. Weisz and U. Wolff, Nucl. Phys. B 359, 221 (1991) 